Annales Academiæ Scientiarum Fennicæ

Series A. I. Mathematica

Volumen 14, 1989, 89-101

\title{
RIEMANN SURFACES WITH THE AD-MAXIMUM PRINCIPLE
}

\author{
Pentti Järvi
}

\section{Introduction}

Let $W$ be an open Riemann surface. We say that $W$ satisfies the (absolute) $A D$-maximum principle if every end $V$ of $W$, i.e., a subregion of $W$ with compact relative boundary $\partial V$, has the property that each function in $A D(\bar{V})$, the class of analytic functions with a finite Dirichlet integral on $\bar{V}=V \cup \partial V$, assumes its supremum on $\partial V$. It is natural to expect that the validity of this principle presumes some sort of weakness of the ideal boundary of $W$. Actually, our main theorem (Theorem 1) asserts that, given any end $V \subset W$ and any $f \in A D(\bar{V})$, the cluster set of $f$ attached to the relative ideal boundary of $V$ is a null-set of class $N_{D}$ in the familiar notation of Ahlfors-Beurling. This result is completely analogous to that of Royden concerning Riemann surfaces which satisfy a similar principle for bounded analytic functions [19].

The interest in the class of surfaces introduced above stems in part from the fact that it contains both $\mathcal{O}_{K D}$ and $\mathcal{O}_{A^{\circ} D}$ (see Chapter 2). In particular, owing to Theorem 1, the boundary theorems of Constantinescu (on $\mathcal{O}_{K D}$-surfaces) and

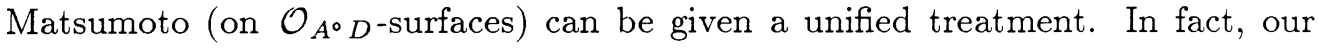
version improves these results in three respects. First, it applies to a wider class of surfaces. Second, instead of $A D$-functions we deal with the larger class of meromorphic functions with a finite spherical Dirichlet integral. Third, we will show that the behavior of the functions at the ideal boundary is not just continuous but even "analytic" in a well justified sense of the word. This in turn makes it possible to draw certain conclusions of an algebraic nature (see Theorem 3 and its corollary).

The main theorem also bears on Royden's version of the Riemann-Roch theorem (on $\mathcal{O}_{K D}$-surfaces). It turns out that, roughly speaking, his result is the pullback of the classical case via a finite sheeted covering map. Furthermore, Theorem 1 entails a Kuramochi-type result concerning the nonexistence of certain meromorphic functions on Riemann surfaces with arbitrary "holes" (Theorem 6). 


\section{The main theorem}

Let $V$ be an end of an open Riemann surface $W$. For the sake of convenience, we always assume that $\partial V$ consists of a finite number of piecewise analytic closed curves. Let $f$ be a nonconstant analytic function on $\bar{V}$. Assuming that $z \in$ $\mathbf{C} \backslash f(\partial V)$, the index of $z$ is defined by

$$
i(z)=(2 \pi)^{-1} \int_{\partial V} d \arg (f(p)-z) .
$$

With suitable interpretation (see [19]), i(z), as well as the valence $v(z)$ of $f$ at $z$ with respect to $\bar{V}$, can be defined also for $z \in f(\partial V)$ and even in such a way that the expression $\delta(z)=i(z)-v(z)$ remains unaltered whenever $V$ is subjected to a compact modification.

Assume now that $W$ satisfies the $A D$-maximum principle, and let $f \in$ $A D(\bar{V})$ be nonconstant. In what follows, our principal aim is to show that $\delta(z) \geq 0$ for all $z \in \mathbf{C}$ and the set $E=\{z \in \mathbf{C} \mid \delta(z)>0\}$ is of class $N_{D}$. The proof is largely based on the ideas of Royden [19]. However, there are some extra problems due to the fact that the class $A D$ is not closed under composition of functions. This state of affairs explains the division of the proof into "topological" and "analytical" parts. More precisely, we first show that $E$ is totally disconnected and then, by means of this preliminary result, that $E$ actually belongs to $N_{D}$. We begin with a simple lemma.

Lemma. Let $K \subset \mathbf{C}$ be a proper continuum with connected complement. Then $\mathbf{C} \backslash K$ carries a nonconstant analytic function $g$ such that both $g$ and $g^{\prime}$ are bounded.

Proof. Obviously we may assume that $K$ is nowhere dense in C. Fix two distinct points $z_{1}, z_{2} \in K$. Denote by $\varphi_{1}$ the restriction to $\mathbf{C} \backslash K$ of a linear fractional mapping that sends $z_{1}$ to 0 and $z_{2}$ to $\infty$. Further, denote by $\varphi_{2}$ some branch of the mapping $z \mapsto z^{1 / 2}, z \in \varphi_{1}(\mathbf{C} \backslash K)$. Pick out a point $z_{3} \in$ $\mathbf{C} \backslash \overline{\varphi_{2}\left(\varphi_{1}(\mathbf{C} \backslash K)\right)}$, and let $\varphi_{3}$ stand for the inversion $z \mapsto 1 /\left(z-z_{3}\right)$. Finally, let $\varphi_{4}$ denote the map $z \mapsto z^{2}$ and $\varphi_{5}$ the map $z \mapsto\left(z-\left(1 / z_{3}\right)^{2}\right)^{2}$. Then $g=\varphi_{5} \circ \varphi_{4} \circ \varphi_{3} \circ \varphi_{2} \circ \varphi_{1}$ is the desired function. $\square$

We return to the function $f \in A D(\bar{V})$ fixed previously. Set $N=$ $\max \{i(z) \mid z \in \mathbf{C}\}$ and let $E_{k}$ denote the closed set $\{z \in \mathbf{C} \mid \delta(z)=i(z)-v(z) \geq$ $k\}, k \in Z$. We claim that $E_{0}=\mathbf{C}$ and $E_{1}(=E)$ is totally disconnected. Observing that $E_{N+1}$ is empty, we assume that $E_{k+1}$ is totally disconnected, $k \leq N$. We show that $E_{k}$ also is totally disconnected provided that it is nowhere dense in C. Set $D_{k}=E_{k} \backslash E_{k+1}$ and let $z \in D_{k}$. Since $\delta$ remains unaltered by the removal from $V$ of a compact set with nice boundary, we may modify $V$ so that $z$ has a neighborhood $U$ such that no point of $U \cap D_{k}$ is assumed (on $\bar{V}$ ) by $f$, while each point of $U \backslash D_{k}$ is assumed (on $V$ ) by $f$. Furthermore, we may arrange 
so that $U \cap f(\partial V)=\emptyset$. If $U \cap D_{k}$ is not totally disconnected, we can obviously find in $U \cap D_{k}$ a proper continuum $K$ with connected complement. Let $g$ be a function described in the preceding lemma. Because $g^{\prime}$ is bounded, $g \circ f$ belongs to $A D(\bar{V})$. However, since $g$ assumes a larger value at some point of $U$ than its maximum in $\mathbf{C} \backslash U, g \circ f$ must take a larger value in $V$ than on $\partial V$. This violates the $A D$-maximum principle for $W$. Thus $U \cap D_{k}$ is totally disconnected. Since this is true for each $z \in D_{k}$ and $E_{k+1}$ is totally disconnected, we conclude that $E_{k}$ is totally disconnected.

Assume then that $D_{k}$ has interior points, and suppose the interior of $D_{k}$ has a boundary point $z$ in the complement of $E_{k+1}$. Modifying $V$ suitably, we can find an open disc $U$ containing $z$ such that $U \cap f(\partial V)=\emptyset, f$ assumes no values in $U \cap D_{k}$ and assumes all values in $U \backslash D_{k}$. Since $U \cap D_{k}$ has interior points, we can find a rational function $g$ whose only pole is in the interior of $U \cap D_{k}$ and which is larger in $U$ than in $\mathbf{C} \backslash U$. Since $g^{\prime}$ is bounded in $\mathbf{C} \backslash\left(U \cap D_{k}\right), g \circ f \in A D(\bar{V})$. However, $|g(f(q))|>\max \{|g(f(p))| \mid p \in \partial V\}$ for each $q \in V$ with $f(q) \in U$. Thus we have again arrived at a contradiction to the $A D$-maximum principle for $W$. It follows that $D_{k}$ has no boundary points in the complement of $E_{k+1}$. But this implies that $D_{k}=\mathbf{C} \backslash E_{k+1}$. In other words, $D_{k}$ is the whole complement of $E_{k+1}$ provided it contains interior points.

Since $f$ is bounded in $\bar{V}, D_{0}$ contains a neighborhood of $\infty$. Therefore, $D_{k}$ has interior points only if $k=0$. Hence $E_{0}=\mathbf{C}$ and the deficiency set $E\left(=E_{1}\right)$ is totally disconnected. We conclude that $f$ has bounded valence: $v(z) \leq N$ for all $z \in \mathbf{C}$.

We are now in a position to establish the definitive result $E \in N_{D}$. The proof is again by induction. Recalling that $E_{N+1}$ is empty, assume $E_{k+1} \in N_{D}$ for some $k \leq N$. Let $z \in D_{k}=E_{k} \backslash E_{k+1}$. As above, we may modify $V$ so that $z$ has a neighborhood $U$ such that $U \cap f(\partial V)=\emptyset$ and no point of $U \cap D_{k}$ is assumed by $f$, while each point of $U \backslash D_{k}$ is assumed by $f$. Suppose there is a compact part, say $F$, of $U \cap D_{k}$ that does not belong to $N_{D}$. Then there is a nonconstant $A D$-function $g$ defined in $\mathbf{C} \backslash F$. As shown previously, $f$ has bounded valence, so that $g \circ f \in A D(\bar{V})$. By the maximum principle, $g$ assumes a larger value at some point of $U$ than its maximum in $\mathbf{C} \backslash U$. Hence $\sup \{|g(f(p))| \mid p \in \bar{V}\}>\max \{|g(f(p))| \mid p \in \partial V\}$, in violation of the $A D$ maximum principle for $W$. We conclude that the compact parts of $U \cap D_{k}$ are of class $N_{D}$. Since this holds for each $z \in D_{k}$, and $E_{k+1} \in N_{D}$ also, we infer that $E_{k} \in N_{D}$. It follows that $E$ belongs to $N_{D}$ as was asserted. We have thereby completed the proof of

Theorem 1. Let $W$ be an open Riemann surface satisfying the $A D$ maximum principle, and let $V$ be an end of $W$. Let $f \in A D(\bar{V})$ be nonconstant. Then $f$ has bounded valence; in fact, $v(z) \leq i(z)$ for each $z \in \mathbf{C}$. Moreover, the deficiency set $E=\{z \in \mathbf{C} \mid v(z)-i(z)<0\}$ is of class $N_{D}$. 


\section{Some consequences}

2.1. We begin with some notation and terminology. By definition, a harmonic function $u$ with a finite Dirichlet integral on a Riemann surface $W$ is in $K D(W)$ if $* d u$ is semiexact, i.e., $\int_{\gamma} * d u=0$ for every dividing cycle $\gamma$ on $W$. If $K D(W)$ reduces to the constants, $W$ is said to belong to $\mathcal{O}_{K D}[21$, p. 132]. Further, $W$ belongs to $\mathcal{O}_{A^{\circ} D}[21$, p. 17] provided every bordered subregion $V$ of $W$, with compact or noncompact border $\partial V$, has the property that the double of $(V, \partial V)$ about $\partial V$ belongs to $\mathcal{O}_{A D}$, the class of surfaces without nonconstant $A D$-functions. Both $\mathcal{O}_{K D}$ and $\mathcal{O}_{A^{\circ} D}$ provide examples of surfaces with the $A D$ maximum principle as appears from

Proposition 1. (a) Every Riemann surface in $\mathcal{O}_{K D} \cup \mathcal{O}_{A^{\circ} D}$ satisfies the $A D$-maximum principle.

(b) Let $W$ be a Riemann surface satisfying the $A D$-maximum principle. Then $W$ belongs to $\mathcal{O}_{A D}$.

Proof. For $W \in \mathcal{O}_{A^{\circ} D}$ the validity of the $A D$-maximum principle is essentially proved in [21, pp. 373-4]. For $W \in \mathcal{O}_{K D}$ the corresponding statement readily follows from assertion IV in [5, p. 1995] (see also [22, p. 254]). Assertion (b) is of course trivial. $\square$

Let $V$ be an end of $W$. Then $M C(V)$ denotes the class of meromorphic functions on $V$ which have a limit at every point of the relative Stoilow ideal boundary $\beta_{V}$ of $V$. Furthermore, $B V(V)$ stands for the class of constants and of meromorphic functions of bounded valence on $V$, while $M D^{*}(V)$ denotes the class of meromorphic functions with a finite spherical Dirichlet integral on $V$. Whenever $f$ is a function of class $M C$, we let $f^{*}$ denote the extension of $f$ to the (relative) ideal boundary.

We first show that the $M D^{*}$-functions behave continuously at the ideal boundary provided $W$ satisfies the $A D$-maximum principle.

Theorem 2. Let $W$ be a Riemann surface satisfying the $A D$-maximum principle, and let $V$ be an end of $W$. Then $M D^{*}(\bar{V})=B V(\bar{V}) \subset M C(\bar{V})$. Furthermore, $f^{*}\left(\beta_{V}\right)$ belongs to $N_{D}$ for every $f \in M D^{*}(V)$.

Proof. Suppose first that $f \in A D(\bar{V})$. By Theorem $1 f$ has bounded valence and the deficiency set $E$ belongs to $N_{D}$. It is not difficult to verify that $\mathrm{Cl}\left(f ; \beta_{V}\right)$, the cluster set of $f$ attached to $\beta_{V}$, is contained in $E$ (details can be found in [10, p. 303]). Since $E$ is totally disconnected, each $\mathrm{Cl}(f ; p)$, the cluster set attached to $p \in \beta_{V}$, must be a singleton, i.e., $f \in M C(\bar{V})$. Finally, $f^{*}\left(\beta_{V}\right) \subset E$ implies $f^{*}\left(\beta_{V}\right) \in N_{D}$.

Now let $f \in M D^{*}(\bar{V})$ be nonconstant. Let $V_{1}, \ldots, V_{n}$ be mutually disjoint subends of $V$ such that $\bar{V} \backslash\left(\bigcup_{i=1}^{n} V_{i}\right)$ is compact and $f$ omits in $\cup_{i=1}^{n} \bar{V}_{i}$ a compact set $E \subset \mathbf{C}$ of positive area measure. By a theorem of Nguyen Xuan Uy [23, Theorem 4.1], we can find a nonconstant analytic function $g$ such that both $g$ 
and $g^{\prime}$ are bounded in $\mathbf{C} \backslash E$. Fix $i \in\{1, \ldots, n\}$. We are going to show that $h=$ $g \circ\left(f \mid \bar{V}_{i}\right)$ belongs to $A D\left(\bar{V}_{i}\right)$. To this end, choose $R>0$ such that $E \subset D(0, R)=$ $\{z \in \mathbf{C}|| z \mid<R\}$. Set $F_{1}=\bar{V}_{i} \cap f^{-1}(D(0, R)), F_{2}=\bar{V}_{i} \cap f^{-1}(\hat{\mathbf{C}} \backslash D(0, R))$ $(\hat{\mathbf{C}}=\mathbf{C} \cup\{\infty\})$ and choose $M>0$ such that $\left|g^{\prime}(z)\right| \leq M$ for $z \in \mathbf{C} \backslash E$. Then

$$
\begin{aligned}
\iint_{F_{1}} d h \wedge * d \bar{h} & =\iint_{F_{1}}\left|g^{\prime}(f(p))\right|^{2} d f \wedge * d \bar{f} \\
& \leq\left(1+R^{2}\right)^{2} M^{2} \iint_{F_{1}} \frac{1}{\left(1+R^{2}\right)^{2}} d f \wedge * d \bar{f} \\
& \leq\left(1+R^{2}\right)^{2} M^{2} \iint_{F_{1}} \frac{1}{\left(1+|f(p)|^{2}\right)^{2}} d f \wedge * d \bar{f}<\infty .
\end{aligned}
$$

Let $\varphi$ denote the mapping $z \mapsto 1 / z, z \in \mathbf{C} \backslash D(0, R)$. Then $g \mid \hat{\mathbf{C}} \backslash D(0, R)=g_{1} \circ \varphi$ with $g_{1}$ analytic in $\overline{D(0,1 / R)}$. Suppose $\left|g_{1}^{\prime}(z)\right| \leq M_{1}$ for $\in \overline{D(0,1 / R)}$. Then

$$
\begin{aligned}
\iint_{F_{2}} d h \wedge * d \bar{h} & =\iint_{F_{2}}\left|g_{1}^{\prime}(\varphi(f(p)))\right|^{2} d(\varphi \circ f) \wedge * \overline{d(\varphi \circ f)} \\
& \leq M_{1}^{2} \iint_{F_{2}} d(\varphi \circ f) \wedge * d \overline{(\varphi \circ f)} \\
& \left.\leq M_{1}^{2}(1+1 / R)^{2}\right)^{2} \iint_{F_{2}} \frac{1}{\left(1+|\varphi(f(p))|^{2}\right)^{2}} d(\varphi \circ f) \wedge * d \overline{(\varphi \circ f)} \\
& \left.=M_{1}^{2}(1+1 / R)^{2}\right)^{2} \iint_{F_{2}} \frac{1}{\left(1+|f(p)|^{2}\right)^{2}} d f \wedge * d \bar{f}<\infty .
\end{aligned}
$$

Thus $h \in A D\left(\bar{V}_{i}\right)$. By Theorem $1, h=g \circ\left(f \mid \bar{V}_{i}\right)$ has bounded valence. Of course, the same is true of $f \mid \bar{V}_{i}$. Since $i \in\{1, \ldots, n\}$ was arbitrary and $\bar{V} \backslash \cup_{i=1}^{n} V_{i}$ is compact, $f$ has bounded valence, too.

Now pick out a point $z_{0} \in \mathbf{C}$ such that the valence of $f$ attains its maximum at $z_{0}$. Choose a small disc $U$ centered at $z_{0}$ such that $f^{-1}(U)$ consists of a finite number of mutually disjoint Jordan domains in $V$. Let $\psi$ stand for the mapping $z \mapsto 1 /\left(z-z_{0}\right)$. Then $\psi \circ f$ is bounded in $\bar{V} \backslash f^{-1}(U)$. This implies that $\psi \circ f \in A D\left(\bar{V} \backslash f^{-1}(U)\right)$. By the first part of the proof, $\psi \circ f \in M C\left(\bar{V} \backslash f^{-1}(U)\right)$ and $(\psi \circ f)^{*}\left(\beta_{V}\right) \in N_{D}$. But then $f \in M C(\bar{V})$ and $f^{*}\left(\beta_{V}\right) \in N_{D}$ also.

The inclusion $B V(\bar{V}) \subset M D^{*}(\bar{V})$ being trivial, the proof is complete. $\square$

Remark. The argument proving the relation $h \in A D(\bar{V})$ also appears in the forthcoming paper [12].

In view of the next theorem we may even claim that the $M D^{*}$-functions admit an analytic extension to the ideal boundary. 
Theorem 3. Let $W$ be a Riemann surface satisfying the $A D$-maximum principle and let $p_{0}$ be a point of $\beta$, the ideal boundary of $W$, such that some end $V \subset W$ with $p_{0} \in \beta_{V}$ carries a nonconstant $M D^{*}$-function. Then there is an end $V_{0} \subset W$ with $p_{0} \in \beta_{V_{0}}$ and an analytic map $\varphi$ of bounded valence from $\bar{V}_{0}$ into the closed unit disc $\bar{D}$ with $\varphi\left(\partial V_{0}\right) \subset \partial \bar{D}$ such that for every $f \in M D^{*}\left(\bar{V}_{0}\right)$ $\left(=B V\left(\bar{V}_{0}\right)\right)$ there is a unique function $g$ meromorphic on $\bar{D}$ so that $f=g \circ \varphi$. In particular, $g \mapsto g \circ \varphi$ is an isomorphism of $M(\bar{D})$, the field of meromorphic functions on $\bar{D}$, onto $M D^{*}\left(\bar{V}_{0}\right)$.

We omit the proof because it is essentially the same as that of $[10$, Theorem $5]$, given in the context of Riemann surfaces with the $A B$-maximum principle. How to treat sets of class $N_{D}$ instead of $N_{B}$ appears from [9, p. 14].

Corollary. Let $W$ be a Riemann surface satisfying the $A D$-maximum principle and let $V \subset W$ be an end. Then $M D^{*}(\bar{V})(=B V(\bar{V}))$ is a field.

Remark. In view of Proposition 1, Theorems 2 and 3 provide improvements of the boundary theorems of Constantinescu ([5, Théorème 1], [6, Theorem], [22, Theorem X 4 C (a)]) and Matsumoto ([15, Theorem 3], [21, Theorem VI 2 C]; see also [12, Theorem 3]). In particular, it follows from Theorem 2 that the requirement that the boundary elements be weak is superfluous in Constantinescu's theorem. This statement is at odds with Remark 2 in [22, p. 265]. It seems that the authors of [22] overlooked the possibility that the class of functions involved in Theorem X 4 C (c) reduces to the constants.

2.2. A global counterpart to the preceding theorem is the following

Theorem 4. Let $W$ be a Riemann surface satisfying the $A D$-maximum principle. Then either

(a) $M D^{*}(W)=B V(W)=\mathbf{C}$, or

(b) $M D^{*}(W)(=B V(W))$ is a field algebraically isomorphic to the field of rational functions on a compact Riemann surface $W_{0}$, which is uniquely determined up to a conformal equivalence. Moreover, the isomorphism is induced by an analytic mapping $\varphi$ of bounded valence from $W$ into $W_{0}$ such that the deficiency set of $\varphi$ (i.e., the set of points in $W_{0}$ not covered maximally) is of class $N_{D}$.

Proof. Suppose that $M D^{*}(W)$ contains a nonconstant function $f$. By Theorem $2, f^{*}(\beta)$ belongs to $N_{D}$. Thus $f^{*}(\beta)$ does not separate the plane. Hence the valence of $f$ is finite and constant in $\hat{\mathbf{C}} \backslash f^{*}(\beta)$; in other words, the deficiency set of $f$ belongs to $N_{D}$. The theorem now follows from [9, Theorem 7]. व

Remark. Suppose, in particular, that $W$ has finite genus. Then $W$ can be taken as the complement of a closed set of class $N_{D}$ on a compact surface $W^{*}$. It is readily shown (see e.g. [9, Lemma 6]) that the elements of $B V(W)$ coincide 
with the restrictions to $W$ of the rational functions on $W^{*}$. Accordingly, the same is true of $M D^{*}(W)$ so that we may take $W_{0}=W^{*}$ in this situation.

The next theorem shows how to find the genus of $W_{0}$ in terms of analytic differentials on $W$. The proof to be given is an adaptation of the argument by Accola [1, pp. 23-24]. As usual, $\Gamma_{a}(W)$ denotes the space of square integrable analytic differentials on $W$, while $\Gamma_{a}^{\prime}(W)$ stands for the subspace of $\Gamma_{a}(W)$ consisting of differentials which are exact outside some compact set (which may depend on the differential).

Theorem 5. Let $W$ be a Riemann surface satisfying the $A D$-maximum principle and suppose $M D^{*}(W)$ contains a nonconstant function. Let $W_{0}$ be the compact Riemann surface described in Theorem 4. Then the genus of $W_{0}$ equals $\operatorname{dim} \Gamma_{a}^{\prime}(W)$.

Proof. Let $\varphi: W \rightarrow W_{0}$ be the mapping given in the preceding theorem. Since the genus of $W_{0}$ equals $\operatorname{dim} \Gamma_{a}\left(W_{0}\right)$, it is enough to show that $\Gamma_{a}^{\prime}(W)=$ $\left\{\varphi^{*} \omega \mid \omega \in \Gamma_{a}\left(W_{0}\right)\right\}$, where $\varphi^{*} \omega$ denotes the pullback of $\omega$ via $\varphi$.

Let $E \subset W_{0}$ denote the deficiency set of $\varphi$; recall that $E$ is of class $N_{D}$. Since $E$ is totally disconnected, we can find an open simply connected neighborhood $U$ of $E$. Fix $\omega \in \Gamma_{a}\left(W_{0}\right)$. Then $\omega \mid U=d f$ with $f$ analytic in $U$. Now $\varphi^{-1}\left(W_{0} \backslash U\right) \subset W$ is compact and $\varphi^{*} \omega \mid \varphi^{-1}(U)=d(f \circ \varphi)$, so that $\varphi^{*} \omega \in \Gamma_{a}^{\prime}(W)$.

Conversely, fix $\omega \in \Gamma_{a}^{\prime}(W)$ not identically zero (if $\Gamma_{a}^{\prime}(W)=\{0\}$, then also $\Gamma_{a}\left(W_{0}\right)=\{0\}$ by the preceding argument). By definition, there is a compact set $K \subset W$ such that $\omega \mid W \backslash K=d f$ for some $f \in A D(W \backslash K)$. Further, let $\omega_{0}$ be a nontrivial meromorphic differential on $W_{0}$ whose poles do not lie in $E$. As above, we can find a compact set $K^{\prime} \subset W$ and a function $g \in A D\left(W \backslash K^{\prime}\right)$ such that $\varphi^{*} \omega_{0} \mid W \backslash K^{\prime}=d g$. We are going to show that the function $\omega / \varphi^{*} \omega_{0}$ belongs to $M D^{*}(W)$.

Fix $p_{0} \in \beta$, the ideal boundary of $W$. Invoking Theorem 3, we can find an end $V_{0} \subset W$ such that $p_{0} \in \beta_{V_{0}}$ and $\bar{V}_{0} \cap\left(K \cup K^{\prime}\right)=\emptyset$ and an analytic mapping $\psi$ from $\bar{V}_{0}$ into $\bar{D}$ such that $f \mapsto f \circ \psi$ is an isomorphism of $M(\bar{D})$ onto $M D^{*}\left(\bar{V}_{0}\right)$. Hence there are functions $f_{0}, g_{0} \in M(\bar{D})$ such that $f \mid \bar{V}_{0}=$ $f_{0} \circ \psi$ and $g \mid \bar{V}_{0}=g_{0} \circ \psi$. Of course, $f_{0}^{\prime} / g_{0}^{\prime}$ also belongs to $M(\bar{D})$, and because $\left(f_{0}^{\prime} / g_{0}^{\prime}\right) \circ \psi=(d f / d g)\left|\bar{V}_{0}=\left(\omega / \varphi^{*} \omega_{0}\right)\right| \bar{V}_{0},\left(\omega / \varphi^{*} \omega_{0}\right) \mid \bar{V}_{0}$ belongs to $M D^{*}\left(\bar{V}_{0}\right)$. By the compactness of $\beta, \omega / \varphi^{*} \omega_{0} \in M D^{*}(W)$, as was asserted.

By Theorem 4, there is a rational function $h_{0}$ on $W_{0}$ such that $\omega / \varphi^{*} \omega_{0}=$ $h_{0} \circ \varphi$. Thus $\omega=\left(h_{0} \circ \varphi\right) \varphi^{*} \omega_{0}=\varphi^{*}\left(h_{0} \omega_{0}\right)$, i.e., $\omega$ is the pullback via $\varphi$ of an analytic differential on $W_{0}$. The proof is complete. $\square$

2.3. Consider now the situation of [17, Section 3] (see also [21, pp. 138144]); in other words, suppose $W$ is an open Riemann surface of class $\mathcal{O}_{K D}$, and let $\mathcal{M}(W)$ denote the class of all meromorphic functions $f$ on $W$ such that $f$ has a finite number of poles and a finite Dirichlet integral over the complement of a neighborhood of its poles. Assume also that $\mathcal{M}(W)$ is nontrivial, i.e., 
contains a nonconstant function. Since $W$ satisfies the $A D$-maximum principle (Proposition 1), each $f \in \mathcal{M}(W)$ is bounded outside a compact subset of $W$. Thus $\mathcal{M}(W)$ constitutes a ring. We maintain that the quotient field of $\mathcal{M}(W)$ is $M D^{*}(W)(=B V(W))$. Indeed, given a nonconstant function $f \in M D^{*}(W)$ choose a point $z_{0} \in \mathbf{C}$ such that the valence of $f$ attains its maximum at $z_{0}$. Then $g=1 /\left(f-z_{0}\right)$ is bounded off a compact subset of $W$ (cf. the proof of Theorem 2). Hence $g \in \mathcal{M}(W)$. Since $f=\left(1+z_{0} g\right) / g$, the assertion follows.

By Theorem 4, there exist a compact Riemann surface $W_{0}$ and an analytic mapping $\varphi$ of a bounded valence from $W$ into $W_{0}$ such that each $f \in M D^{*}(W)$ admits a representation

$$
f=g \circ \varphi
$$

where $g$ is a rational function on $W_{0}$. Clearly, $f \in \mathcal{M}(W)$ if and only if $g$ is a rational function on $W_{0}$ whose poles lie outside the deficiency set of $\varphi$. It follows that the problem of whether there is a function in $\mathcal{M}(W)$ which is a multiple of a given divisor and has a given principal part can be decided in terms of analytic objects on $W_{0}$. In this sense, Theorem 2 in [17, p. 47] ([21, Theorem II 16 I]) can be regarded as the pullback via the map $\varphi$ of the classical Riemann-Roch theorem. Of course, the rigidity of the class $\mathcal{M}(W)$, as evidenced in $(*)$, imposes severe limitations on potential singularities for elements of $\mathcal{M}(W)$. In particular, $\mathcal{M}(W)$ separates points of $W$ if and only if $W$ has finite genus. As observed previously, $W$ is then the complement of a set of class $N_{D}$ on a compact Riemann surface.

Remark 1. In his paper [1] Accola discusses more broadly generalizations of some classical theorems from the point of view of Heins' composition theorem [8], which is a special case of Theorem 4.

Remark 2. A frequent substitute for the field of rational functions is the class of quasirational functions in the sense of Ahlfors [3. p. 316]. In the present situation a function is quasirational if and only if it belongs to $M D^{*}(W)$ and is bounded away both from 0 and from $\infty$ outside some compact subset of $W$.

2.4. Let $U_{S}$ denote the class of open Riemann surfaces whose ideal boundary contains a point of positive harmonic measure [21, p. 385]. Suppose that $W$ belongs to $U_{S}$ and satisfies the $A D$-maximum principle, and let $K$ be an arbitrary compact set in $W$ with connected complement. Let $f \in M D^{*}(W \backslash K)$. By Theorem 2, $f$ admits a continuous extension to the ideal boundary of $W$. On the other hand, Theorem X $4 \mathrm{C}$ (c) in [22] implies that $f$ must be constant. Thus, denoting by $\mathcal{O}_{M D^{*}}$ the class of Riemann surfaces without nonconstant $M D^{*}$ functions, we obtain the following theorem, which contains [21, Theorem VI 5 B] and [12, Corollary to Theorem 3]. 
Theorem 6. Let $W$ be a Riemann surface which satisfies the $A D$-maximum principle and belongs to $U_{S}$, and let $K$ be an arbitrary compact set in $W$ with connected complement. Then $W \backslash K \in \mathcal{O}_{M D^{*}}$.

Familiar instances of $U_{S}$-surfaces are furnished by the interesting class $\mathcal{O}_{H D} \backslash$ $\mathcal{O}_{G}$, where $\mathcal{O}_{H D}$ is the class of Riemann surfaces without nonconstant Dirichlet bounded harmonic functions and $\mathcal{O}_{G}$ the class of parabolic surfaces. Recall that the ideal boundary of each $W \in \mathcal{O}_{H D} \backslash \mathcal{O}_{G}$ contains exactly one point of positive harmonic measure. Since every surface in $\mathcal{O}_{H D}$ also satisfies the $A D$-maximum principle (for $\mathcal{O}_{H D} \subset \mathcal{O}_{K D}$ ), we have the original version of Kuramochi [14, Theorem 1], [21, Corollary to Theorem III 5I].

Corollary. Let $W \in \mathcal{O}_{H D} \backslash \mathcal{O}_{G}$ and let $K$ be an arbitrary compact set in $W$ with connected complement. Then $W \backslash K \in \mathcal{O}_{A D}$.

\section{Characterizations of Riemann surfaces with the $A D$-maximum principle}

3.1. It is clear that a Riemann surface of finite genus satisfies the $A D$ maximum principle if and only if it belongs to $\mathcal{O}_{A D}$. On the other hand, it is known that for these surfaces $\mathcal{O}_{A D}=\mathcal{O}_{K D}$ [21, Theorem II $\left.14 \mathrm{D}\right]$. However, in the general case the inclusions given in Proposition 1 are strict. The next theorem gives some criteria to recognize surfaces with the $A D$-maximum principle. In particular, we will show that it suffices to impose the maximum principle on the bounded $A D$-functions. Given an open Riemann surface $W$ and an end $V \subset W$, we set $A B D(V)=\{f \mid f$ is bounded in $V$ and $f \in A D(V)\}$ and say that $W$ satisfies the $A B D$-maximum principle if $\max \{|f(p)| \mid p \in \partial V\}=\sup \{|f(p)| \mid p \in \bar{V}\}$ for each end $V \subset W$ and for each $f \in A B D(\bar{V})$.

Theorem 7. Let $W$ be an open Riemann surface. Then the following statements are equivalent:

(1) $W$ satisfies the $A D$-maximum principle.

(2) $W$ satisfies the $A B D$-maximum principle.

(3) $M D^{*}(\bar{V}) \subset B V(\bar{V})$ for every end $V \subset W$.

(4) For every end $V \subset W$ and for every $f \in M D^{*}(\bar{V})$ the cluster set of $f$ attached to the relative ideal boundary of $V$ is totally disconnected.

Proof. The implications (1) $\Rightarrow(3)$ and $(1) \Rightarrow(4)$ are direct consequences of Theorem 2. Also, $(4) \Rightarrow(3)$ is immediate by observing that the valence function is finite and constant in every component of $\hat{\mathbf{C}} \backslash\left(f(\partial V) \cup \mathrm{Cl}\left(f ; \beta_{V}\right)\right)$. The implication $(1) \Rightarrow(2)$ being trivial, there remains to be proved $(3) \Rightarrow(2)$ and $(2) \Rightarrow$ (1).

$(3) \Rightarrow(2)$ : Suppose there is an end $V \subset W$ and an $A B D$-function $f$ on $\bar{V}$ with $\max \{|f(p)| \mid p \in \partial V\}<\sup \{|f(p)| \mid p \in \bar{V}\}$. We may assume that 
$\sup \{|f(p)| \mid p \in \bar{V}\}=1$. We maintain that $A D(\bar{V})$ contains a function of unbounded valence. If $f \notin B V(\bar{V})$, there is nothing to prove. Otherwise pick out a sequence of points $\left(z_{n}\right)$ in $f(V)$ such that

$$
\sum_{n=1}^{\infty} \log \left(\frac{1}{1-\left|z_{n}\right|}\right)^{-1 / 2}<\infty .
$$

By a result of Carleson [4, Theorem 1], there is a nonconstant $A D$-function $g$ in the open unit disc such that $g\left(z_{n}\right)=0$ for each $n$. Since $f$ has bounded valence, $g \circ f \in A D(\bar{V})$, while $g \circ f \notin B V(\bar{V})$. The implication follows.

$(2) \Rightarrow(1)$ : Suppose that $W$ satisfies the $A B D$-maximum principle, and let $V$ be an end of $W$. If suffices to show that $A D(\bar{V})$ contains no unbounded function. Assume $A D(\bar{V})$ contains one, say $f$, and fix $R>0$ such that $f(\partial V) \subset D(0, R)$ ( = the open disc of radius $R$ centered at 0 ). Assume first that the interior of $(\hat{\mathbf{C}} \backslash D(0, R)) \backslash f(V)$ is nonempty. Then we can find a point $z_{0}$ in this interior and a positive $r$ such that $D\left(z_{0}, r\right) \cap f(V) \neq \emptyset$ and $D\left(z_{0}, r\right) \cap D(0, R)=\emptyset$. It follows that $1 /\left(f-z_{0}\right)$ belongs to $A B D(\bar{V})$ and takes a larger value in $V$ than on $\partial V$. This contradicts the $A B D$-maximum principle for $W$. Assume then that $f(V)$ is dense in $\hat{\mathbf{C}} \backslash \overline{D(0, R)}$. Since $f \in A D(\bar{V}), f$ omits in $\bar{V}$ a compact set $E \subset \mathbf{C} \backslash \overline{D(0, R)}$ of positive area measure. Invoking [23, Theorem 4.1], we find a nonconstant analytic function $g$ such that $g$ and $g^{\prime}$ are bounded in $\mathbf{C} \backslash E$. Then $g \circ f \in A B D(\bar{V})$. The set $f(V)$ being dense in $\hat{\mathbf{C}} \backslash \overline{D(0, R)}, g \circ f$ assumes a larger value at some point of $V$ than its maximum on $\partial V$. Thus we have again arrived at a contradiction to the $A B D$-maximum principle for $\mathrm{W}$.

Remark. In view of Theorem 2 and Corollary to Theorem 3 one may ask whether the condition $M D^{*}(\bar{V}) \subset M C(\bar{V})$ or the field property of $M D^{*}(\bar{V})$ implicates the validity of the $A D$-maximum principle. Cf. also [11, Theorems 7 and 2]. Unfortunately, we have not been able to answer these questions.

3.2. Suppose $V$ is an end of a Riemann surface satisfying the $A D$-maximum principle. If the genus of $V$ is infinite, $A D(\bar{V})$ fails to separate points of $\bar{V}$; this is immediate by Theorem 3. In other words, in case $A D(\bar{V})$ separates points, $V$ can be taken as the complement on a finite Riemann surface of a closed set of class $N_{D}$. Actually, in order to establish this result one need not the full force of the $A D$-maximum principle as shown by

Theorem 8. Let $W$ be an open Riemann surface, and let $V \subset W$ be an end such that $\partial V$ consists of a finite number of closed analytic curves. Suppose that $A D(\bar{V})$ separates the points of $\bar{V}$ and for each $f \in A D(\bar{V}) \max \{|f(p)| \mid$ $p \in \partial V\}=\sup \{|f(p)| \mid p \in \bar{V}\}$. Then there exist a finite Riemann surface $V^{*}$ and a compact set $E \subset V^{*}$ of class $N_{D}$ such that $V$ is conformally equivalent to $V^{*} \backslash E$. Further, $V^{*}$ is uniquely determined up to a conformal equivalence. 
Proof. The assertion is an easy consequence of a theorem by Royden. Namely, by [20, Theorem 3] $V$ has finite genus. Therefore, $V$ can be taken as a subdomain of a compact surface $W_{0}$ such that $W_{0} \backslash V$ consists of a finite number of mutually disjoint closed discs $U_{1}, \ldots, U_{n}$ corresponding the components of $\partial V$ and of a closed set $E$. Set $V^{*}=V \cup E$. Assuming that $E$ fails to be of class $N_{D}$, we can find a nonconstant function $f$ in $A D\left(W_{0} \backslash E\right)$ [21, Theorem I $\left.8 \mathrm{E}\right]$. By the maximum principle $\max \left\{|f(p)| \mid p \in \bigcup_{i=1}^{n} \partial U_{i}\right\}<\sup \{|f(p)| \mid p \in V\}$, contrary to the assumption. The uniqueness of $V^{*}$ is obtained by observing that sets of class $N_{D}$ are removable singularities for conformal mappings. $\square$

Remark. Wermer [24] has proved a similar result about Riemann surfaces satisfying the corresponding maximum principle for bounded analytic functions.

\section{Concluding remarks}

Needless to say, the validity of the $A D$-maximum principle is preserved under conformal mappings. More generally, given two Riemann surfaces $W$ and $W^{\prime}$ and a proper analytic mapping $W \rightarrow W^{\prime}, W$ satisfies the $A D$-maximum principle if and only if $W^{\prime}$ does. Also it is clear from the very definition that validity of the $A D$-maximum principle, unlike belonging to $\mathcal{O}_{A D}$, is a property of the ideal boundary (see $[21$, p. 54]).

Riemann surfaces with small boundary are close to being maximal. For instance, it is known that surfaces of class $\mathcal{O}_{K D}$ or $\mathcal{O}_{A^{\circ} D}$ are essentially maximal, i.e., they cannot be realized as nondense subdomains of other surfaces. For $\mathcal{O}_{K D}$ this result is due to Jurchescu (see [22, p. 270]) and for $\mathcal{O}_{A^{\circ} D}$ to Qiu Shuxi [16, Theorem 3]. On the other hand, one can exhibit Riemann surfaces which are essentially extendable and satisfy the $A D$-maximum principle; take, for example, the construction by Heins [7, pp. 298-299] modified in an obvious way. However, provided that a surface also carries enough locally defined $M D^{*}$-functions, it is essentially maximal; what is more, the ideal boundary is absolutely disconnected (see [22, pp. 240 and 270]).

Proposition 2. Let $W$ be a Riemann surface which satisfies the $A D$ maximum principle and let $\beta$ be the ideal boundary of $W$. Suppose that for every point $p \in \beta$ there is an end $V \subset W$ with $p \in \beta_{V}$ such that $M D^{*}(V)$ contains a nonconstant function. Then $\beta$ is absolutely disconnected.

Proof. Fix $p_{0} \in \beta$. By Theorem 3 we can find an end $V \subset W$ with $p_{0} \in \beta_{V}$ and a function $f \in B V(\bar{V})=M D^{*}(\bar{V})$ such that $f(V) \subset D$, the open unit disc, and $f(\partial V) \subset \partial D$. Since $f^{*}\left(\beta_{V}\right)$ belongs to $N_{D}$ (Theorem 2), and the mapping $p \mapsto f(p), V \backslash f^{-1}\left(f^{*}\left(\beta_{V}\right)\right) \rightarrow D \backslash f^{*}\left(\beta_{V}\right)$ is proper, we can apply [13, Theorem 1]. Thus $\beta_{V}$ is absolutely disconnected. Since $p_{0} \in \beta$ was arbitrary, the proposition follows. $\square$ 


\section{References}

[1] Accola, R.: Some classical theorems on open Riemann surfaces. - Bull. Amer. Math. Soc. 73, 1967, 13-26.

[2] Ahlfors, L., and A. Beurling: Conformal invariants and function-theoretic null-sets. - Acta Math. 83, 1950, 101-129.

[3] Ahlfors, L., and L. Sario: Riemann surfaces. - Princeton University Press, Princeton, N.J., 1960.

[4] Carleson, L.: On the zeros of functions with bounded Dirichlet integrals. - Math. Z. 56, 1952, 289-295.

[5] Constantinescu, C.: Sur le comportement d'une fonction analytique à la frontière idéale d'une surface de Riemann. - C. R. Acad. Sci. Paris 245, 1957, 1995-1997.

[6] Constantinescu, C.: On the behavior of analytic functions at boundary elements of Riemann surfaces. - Rev. Roumaine Math. Pures Appl. 2 (Hommage à S. Stö̈low pour son $70^{\mathrm{e}}$ anniversaire), 1957, 269-276 (Russian).

[7] Heins, M.: Riemann surfaces of infinite genus. - Ann. of Math. 55, 1952, 296-317.

[8] Heins, M.: On certain meromorphic functions of bounded valence. - Rev. Roumaine Math. Pures Appl. 2 (Hommage à S. Stoïlow pour son 70 anniversaire), 1957,263-267.

[9] Järvi, P.: Removability theorems for meromorphic functions. - Ann. Acad. Sci. Fenn. Ser. A I Math. Dissertationes 12, 1977, 1-33.

[10] J̈̈RVI, P.: Meromorphic functions on certain Riemann surfaces with small boundary. Ann. Acad. Sci. Fenn. Ser. A I Math. 5, 1980. 301-315.

[11] J̈̈RVI, P.: On meromorphic functions continuous on the Stoillow boundary. - Ann. Acad. Sci. Fenn. Ser. A I Math. 9, 1984, 33-48.

[12] J̈̈Rvi, P.: On the continuation of meromorphic functions. - Ann. Acad. Sci. Fenn. Ser. A I Math. 12, 1987, 177-184.

[13] Jurchescu, M.: A maximal Riemann surface. - Nagoya Math. J. 20, 1962, 91-93.

[14] Kuramochi, Z.: On the behavior of analytic functions on abstract Riemann surfaces. Osaka Math. J. 7, 1955, 109-127.

[15] Matsumoto, K.: Analytic functions on some Riemann surfaces II. - Nagoya Math. J. 23, 1963, 153-164.

[16] QIU, S.: The essential maximality and some other properties of a Riemann surface of $\mathcal{O}_{A^{\circ} D}$. - Chin. Ann. Math. Ser. B 6, 1985, 317-323.

[17] Royden, H.L.: The Riemann-Roch theorem. - Comment. Math. Helv. 34, 1960, 37-51.

[18] Royden, H.L.: On a class of null-bounded Riemann surfaces. - Comment. Math. Helv. $34,1960,52-66$.

[19] Royden, H.L.: Riemann surfaces with the absolute AB-maximum principle. - Proceedings of the Conference on Complex Analysis, Minneapolis, 1964, edited by A. Aeppli, E. Calabi and H. Röhrl. Springer-Verlag, Berlin-Heidelberg-New York, 1965, 172175 .

[20] Royden, H.L.: Algebras of bounded analytic functions on Riemann surfaces. - Acta Math. $114,1965,113-142$.

[21] SaRio, L., and M. NAKaI: Classification theory of Riemann surfaces. - Die Grundlehren der mathematischen Wissenschaften 164. Springer-Verlag, Berlin-Heidelberg-New York, 1970. 
[22] Sario, L., and K. Oikawa: Capacity functions. - Die Grundlehren der mathematischen Wissenschaften 149. Springer-Verlag, Berlin-Heidelberg-New York, 1969.

[23] Uy, N.X.: Removable sets of analytic functions satisfying a Lipschitz condition. - Ark. Mat. 17, 1979, 19-27.

[24] Wermer, J.: The maximum principle for bounded functions. - Ann. of Math. 69, 1959, 598-604.

University of Helsinki

Department of Mathematics

Hallituskatu 15

SF-00100 Helsinki

Finland

Received 4 December 1987 